Chirurg 2014 $\cdot 85: 112-116$

DOI 10.1007/s00104-013-2593-x

Online publiziert: 18. Januar 2014

๑) Springer-Verlag Berlin Heidelberg 2014

A. Kohler · G. Beldi

Universitätsklinik für Viszerale Chirurgie und Medizin, Inselspital, Bern

\title{
Rezidiv nach Hernienplastik: Komplikation oder natürlicher Verlauf?
}

divhernie können die beschriebenen Kriterien rein klinisch oft nicht sicher überprüft werden und sind deshalb durch apparative Diagnostik wie Sonographie, Computertomographie oder Laparoskopie zu objektivieren. Die Hernienrezidive lassen sich anhand der anatomischen Kriterien und der Symptome in drei Gruppen einteilen:

\section{Klinisch relevantes Rezidiv}

Ein klinisch relevantes Rezidiv liegt vor, wenn die anatomischen Kriterien erfüllt sind und zusätzlich Symptome wie Schmerzen oder Einklemmungsbeschwerden auftreten. Analog den Kriterien zur primären Operation einer Hernie ist beim Vorliegen dieser Kriterien die Indikation zur Operation gegeben.

\section{Klinisch irrelevantes Rezidiv}

Diese Situation liegt vor, wenn durch klinische und/oder radiologische Untersuchungen ein Rezidiv im Sinne der anatomischen Definition gefunden wird, der Patient diesbezüglich aber beschwerdefrei ist. In dieser Situation besteht keine absolute Indikation zu einer erneuten Operation.

\section{„Pseudorezidiv"}

gnostiziert, sofern die gleichen Kriterien einer primären Hernie mit Bruchring, Bruchsack und Bruchinhalt vorliegen. Trotz dieser einfachen theoretischen Definition ist die Diagnosestellung eines Rezidivs im Alltag komplexer. Bei der Rezi-
Bei Persistenz von subjektiv wahrgenommenen Symptomen und fehlendem Nachweis einer Hernie gemäß anatomischer Definition liegt ein Pseudorezidiv vor. Das Vorliegen eines Pseudore- zidives wird als dreifach häufiger als das Auftreten eines echten Rezidivs beschrieben [27]. Das Beschwerdebild umfasst das Bulging, kosmetische Probleme und chronische Schmerzen.

Bulging ist ein typisches Phänomen nach laparoskopischer Therapie großer Narbenhernien.

Weil bei der laparoskopischen Operation die Bauchwand nicht rekonstruiert wird, kann es bereits früh postoperativ zu einem gewissen Hervortreten des Netzes durch die große Bruchpforte kommen, ohne dass dabei erneut eine Engstelle oder ein eigentlicher Bruch entsteht. Der Patient kann sich aufgrund der Unregelmäßigkeit der Bauchwand kosmetisch gestört fühlen. In unserem Erfahrungsbereich trat ein Bulging nach laparoskopischem Repair von großen Narbenhernien bei $17 \%$ der Patienten auf [16]. Eine ähnliche Symptomatik mit Schwellung ohne eigentliche Hernie ergibt sich beim Auftreten eines postoperativen Seroms oder Hämatoms im Operationsgebiet.

Auch chronische postoperative Schmerzen werden vom Patienten eventuell als ein Rezidiv der Hernie empfunden, obwohl anatomisch gesehen keine Hernie mehr besteht. Die Inzidenz chronischer postoperativer Schmerzen nach Herinenoperation wird in der Literatur sehr unterschiedlich angegeben. Dies beruht auf der Tatsache, dass die Art sowie der Zeitpunkt der Erhebung sehr unterschiedlich gewählt werden [21]. In Studien, in denen chronische Leistenschmer- 
zen ein Jahr postoperativ nach Inguinalhernienrepair durch Befragung des Patienten mittels Fragebogen erhoben wurden, ergeben sich Häufigkeiten um 5\% $[5,20]$. Es scheint, als ob diese Schmerzen seltener nach laparoskopischem Hernienrepair auftreten [6]. Dies erklärt sich am ehesten dadurch, dass die Schmerzen durch Nervenirritation durch Fremdmaterial im Bereich des Inguinalkanals entstehen. So kommt durch die Lage des Netzes im Inguinalkanal bei der Hernienoperation nach Lichtenstein das Netz eher in Kontakt mit dem N. ilioinguinalis und dem R. genitalis des N. genitofemoralis als dies bei den laparoskopischen Techniken der Fall ist.

\section{Erhebung von Rezidivraten}

Die objektive Beurteilung der Rezidivrate ist durch verschiedene Faktoren erschwert. So spielen nebst den unterschiedlichen Definitionen von Hernienrezidiven die Art der Untersuchung und die Dauer des Follow-ups eine erhebliche Rolle und machen den Vergleich von Zahlen in der Literatur problematisch [12].

\section{》) Die tatsächliche Rezidivrate wird unterschätzt}

Eine objektive Abbildung der Rezidivproblematik erfordert eine genügend lange Beobachtungszeit. Zudem muss die korrekte Nachuntersuchung eine klinische Untersuchung einschließen. Schriftliche oder telefonische Befragungen genügen nicht, um die Rezidivrate korrekt zu beurteilen, da die Patienten nicht zwischen einem echten und einem Pseudorezidiv unterscheiden können. Zudem entgehen klinisch irrelevante Rezidive ohne körperliche Untersuchung der Erfassung vollständig. Die Rate an Reoperationen bei Verdacht auf Rezidivhernie genügt auch nicht, da die Indikation für eine erneute Operation auch bei chronischen Schmerzen oder Pseudorezidiven gestellt wird und klinisch irrelevante Rezidive bei dieser Art Betrachtung vollständig untergehen. Des Weiteren lassen viele Patienten sich beim Auftreten eines erneuten Rezidivs nicht ein zweites Mal operieren [14].
Somit wird die echte Rezidivrate sowohl bei Nachuntersuchungen ohne klinische Untersuchung wie auch bei der Quantifizierung von Reoperationen unterschätzt. Nur eine Nachuntersuchung mit ärztlicher klinischer Untersuchung einer möglichst vollständigen Population erlaubt eine adäquate Beurteilung der echten Rezidivrate nach Hernienoperationen.

\section{Standortbestimmung}

\section{Aktuelle Rezidivraten}

bei Inguinalhernien

In den letzten Jahren wurden mehrere größere Studien zur Gegenüberstellung von laparoskopischem und offenem Inguinalhernienrepair mit Netz durchgeführt. Es wurden Rezidivraten im Bereich von $1-5 \%$ für beide Techniken beschrieben $[2,10,18]$. Metaanalysen zeigten tendenziell höhere Rezidivraten mittels endoskopischer total extraperitonealer (TEP) und transabdominal präperitonealer Plastik (TAPP) verglichen mit der offenen Technik nach Lichtenstein [19, 23]. Wobei die laparoskopischen Techniken mit einer signifikant niedrigeren Inzidenz chronischer Schmerzen [19], einer kürzeren Hospitalisationszeit und einer schnelleren Erholung nach der Operation im Vergleich zu den offenen Verfahren einhergehen.

Große Fallserien in spezialisierten Hernienkliniken zeigen jedoch, dass sowohl Rezidiv- als auch Komplikationsrate bei offenen wie laparoskopischen Techniken niedriger sind als in randomisierten Studien. Sowohl für die Operation nach Shouldice [11], Lichtenstein [17] und die TAPP [4] wurden schon vor Jahren Daten publiziert, die zeigen dass die Rezidivraten in hoch spezialisierten Institutionen unter $1 \%$ gehalten werden können. Jedoch muss angemerkt werden, dass in diesen Studien das Follow-up teilweise nicht für die gesamte Studienpopulation systematisch durchgeführt wurde.

\section{$>$ Der Zeitpunkt des Auftretens der Hernienrezidive hängt von der Technik der Primäroperation ab.}

Beim Vergleich verschiedener Methoden ist zu beachten, dass der Zeitpunkt des Auftretens von Langzeitrezidiven je nach Operationstechnik unterschiedlich sein kann [18]. So treten bei netzbasierten Operationstechniken die Rezidive eher $\mathrm{zu}$ einem frühen postoperativen Zeitpunkt auf, einmal eingewachsene Netze scheinen im Verlauf ihre Stabilität zu behalten. Die Ursache eines Hernienrezidivs nach Implantation eines Kunststoffnetzes ist somit eher chirurgisch-technischer Natur. Bei Techniken ohne Netz entwickeln sich Rezidive auch zu späteren Zeitpunkten. Das bei dieser Operation rekonstruierte körpereigene Gewebe kann auch nach Jahren wieder eine neue Schwäche entwickeln ([3, 28], - Abb. 1). Hier ist die Unterscheidung zwischen Komplikation und natürlichem Verlauf unscharf. Rezidive welche nach über 5 Jahren nach Inguinalhernienplastik auftreten stellen wahrscheinlich den natürlichen Verlauf dar und sind weniger als Komplikation zu definieren.

\section{Aktuelle Rezidivraten \\ bei Narbenhernien}

Die Rezidivrate nach Narbenhernienoperationen wird in der Literatur sowohl für die laparoskopische als auch die offene Operation im Bereich von 15-20\% beschrieben $[1,9,13,24]$. Bezüglich der Rezidivrate konnten randomisierte kontrollierte Studien für keine der zwei Techniken eine generelle Überlegenheit nachweisen $[9,13]$. Es konnte gezeigt werden, dass ein Herniendurchmesser über $5 \mathrm{~cm}$ ein unabhängiger Risikofaktor für die Entwicklung eines Hernienrezidivs darstellt [8].

\section{Komplikation oder natürlicher Verlauf?}

Mit dem Ziel die chirurgische Technik weiter zu verbessern, soll eine sehr niedrige Rezidivrate bei geringen Nebenerscheinungen wie Schmerzen angestrebt werden. Aus diesem Grund darf sich der Chirurg nicht mit der Feststellung zufrieden geben, dass es sich u. U. um einen natürlichen Verlauf handelt, sondern soll primär von einer Komplikation ausgehen. 
Trotzdem sind Risikoprofile für ein Hernienrezidiv sehr unterschiedlich. Im Falle eines sehr hohen Risikos kann man u. U. effektiv von einem natürlichen Verlauf sprechen. Insbesondere dann, wenn dieselben Risikofaktoren beim Rezidiv wie bei der Primäroperation relevant sind.

Zur besseren Beurteilung der Wahrscheinlichkeit ein Rezidiv zu entwickeln, sind patientenbezogene und technische Risikofaktoren zu diskutieren. Die Evidenz diesbezüglich ist jedoch ungenügend. Insbesondere fehlen prospektive Erhebungen, bei welchen zu Beginn der Studie potenzielle Risikofaktoren für eine primäre Hernie und für ein Hernienrezidiv definiert werden und anhand einer klar umschriebenen Kohorte überprüft werden. Aus diesem Grunde bleibt die Frage, ob sich Risikofaktoren für die primäre Entwicklung einer Hernie mit denen für das Hernienrezidiv decken, ungeklärt.

Dennoch lässt sich das Risiko für ein Hernienrezidiv an retrospektiven Studien abschätzen. Es hängt insbesondere von der gewählten Technik, von der Art der Hernie und von Risikofaktoren des $\mathrm{Pa}$ tienten $\mathrm{ab}$.

\section{Risikofaktor chirurgische Technik}

Insbesondere die Einführung von Kunststoffnetzen hat wesentlich zur Senkung der Rezidivrate beigetragen sowohl in der offenen als auch in der laparoskopischen Chirurgie. Die Einführung von Kunststoffnetzen hat die laparoskopische Operation von Inguinal- und Narbenhernien erst möglich gemacht. Die Reduktion von Rezidiven mit Kunststoffnetzen zeigt sich für spezialisierte und nichtspezialisierte Institutionen und wird robuster bei den Langzeitresultaten [3]. Die aktuellen Bestrebungen in der Hernienchirurgie zielen häufig auf die Reduktion chronischer Schmerzen durch Verwendung leichtgewichtiger Netze oder alternativer Fixationsmethoden. Bei all diesen Bestrebungen darf die Rezidivrate nicht außer Acht gelassen werden. Problematisch erscheint hier vor allem, dass die Patientenpopulation in vielen Studien so klein gewählt wurde, dass zwar eine mögliche Reduktion der Schmerzen, nicht jedoch eine Veränderung der Rezidivrate erkannt werden kann.

Chirurg 2014 - 85:112-116 DOI 10.1007/s00104-013-2593-x

c) Springer-Verlag Berlin Heidelberg 2014

\section{A. Kohler · G. Beldi \\ Rezidiv nach Hernienplastik: Komplikation oder natürlicher Verlauf?}

\section{Zusammenfassung}

Die Rezidivhäufigkeit nach Hernienplastik ist ein Qualitätsmerkmal der chirurgischen Technik. Für eine korrekte Beurteilung der Rezidivrate muss zwischen einem klinisch relevanten, klinisch irrelevanten und einem Pseudorezidiv unterschieden werden. Bei der chirurgischen Therapie von Inguinalhernien werden mit den heutigen Techniken mittels laparoskopischer oder offener Netzeinlage sehr niedrige Rezidivraten erreicht. Somit soll bei einem Rezidiv nach einer Inguinalhernienoperation von einer chirurgischen Komplikation ausgegangen werden. Im Gegensatz zur Inguinalhernie liegt die Rezidivrate bei der Operation großer Narbenhernien trotz stetiger Optimierung der Technik weiterhin über $10 \%$. Um das Rezidivrisiko abzuschätzen, müssen nebst der Größe und Lokalisation der
Hernie technische und patientenspezifische Aspekte beurteilt werden. Je nach Risikoprofil kann das Hernienrezidiv in seltenen Fällen dem natürlichen Verlauf entsprechen.

Im Allgemeinen werden Hernienrezidive als Abweichung von der Norm wahrgenommen und können von der Indikation, Wahl der Operation und der chirurgischen Technik und Taktik abhängen. Somit soll der Chirurg ein postoperatives Rezidiv in erster Linie als Komplikation und nicht als natürlichen Verlauf ansehen, mit dem Ziel die Chirurgie kontinuierlich zu verbessern.

\section{Schlüsselwörter}

Inguinalhernie - Narbenhernie · Rezidivrate . Komplikationsrate $\cdot$ Risikofaktoren

\section{Recurrence after hernia surgery: complication or natural course?}

\section{Abstract}

Recurrence after hernia surgery remains a relevant measure of surgical quality. In order to correctly assess the recurrence rate the surgeon needs to distinguish clinical relevant recurrence, clinically irrelevant recurrence and pseudo-recurrence. Current surgical techniques for inguinal hernia repair using mesh are associated with a low rate of recurrence. Thus, recurrence after inguinal hernia surgery is seen as a surgical complication. Conversely, the recurrence rate for large incisional hernias remains above $10 \%$ and in rare cases a recurrence might represent the natural course.
Recurrent hernia can depend on the indication, choice of operation and surgical technique. Therefore, postoperative hernia recurrence probably does not represent the natural course but rather a surgical complication that needs to be prevented by an optimized surgical technique.

\section{Keywords}

Inguinal hernia $\cdot$ Incisional hernia .

Recurrence rate - Complication rate .

Risk factors

\section{Risikofaktor Hernientypus}

Ätiologisch unterschiedlich entstandene Hernien können bezüglich der Rezidivrate nicht miteinander verglichen werden. Die den Bruchsack bildenden anatomischen Strukturen sind zwischen diesen Hernien unterschiedlich, was die Möglichkeit der Versorgung als auch die Festigkeit postoperativ beeinflusst. Insbesondere Narbenhernien weisen eine sehr hohe Rezidivrate auf und können diesbezüglich nicht mit anderen Hernien verglichen werden. Im Gegensatz zu Inguinalhernien ist sowohl Größe als auch Lokalisation bei Narbenhernien sehr varia- bel und kann somit sehr über die Wahrscheinlichkeit, ein Rezidiv zu entwickeln, entscheiden [8].

\section{Risikofaktor Patient}

Das zusätzliche Fettgewebe adipöser Patienten ist $z$. T. intraabdominal lokalisiert und führt zu einem erhöhten intraabdominellen Druck, was wiederum das Entstehen eines Hernienrezidivs begünstigt [22].

Ähnlich ist der Zusammenhang zwischen Rauchen und einer schlechten Wundheilung und dem Auftreten von Narbenhernien nachgewiesen [25, 26]. 


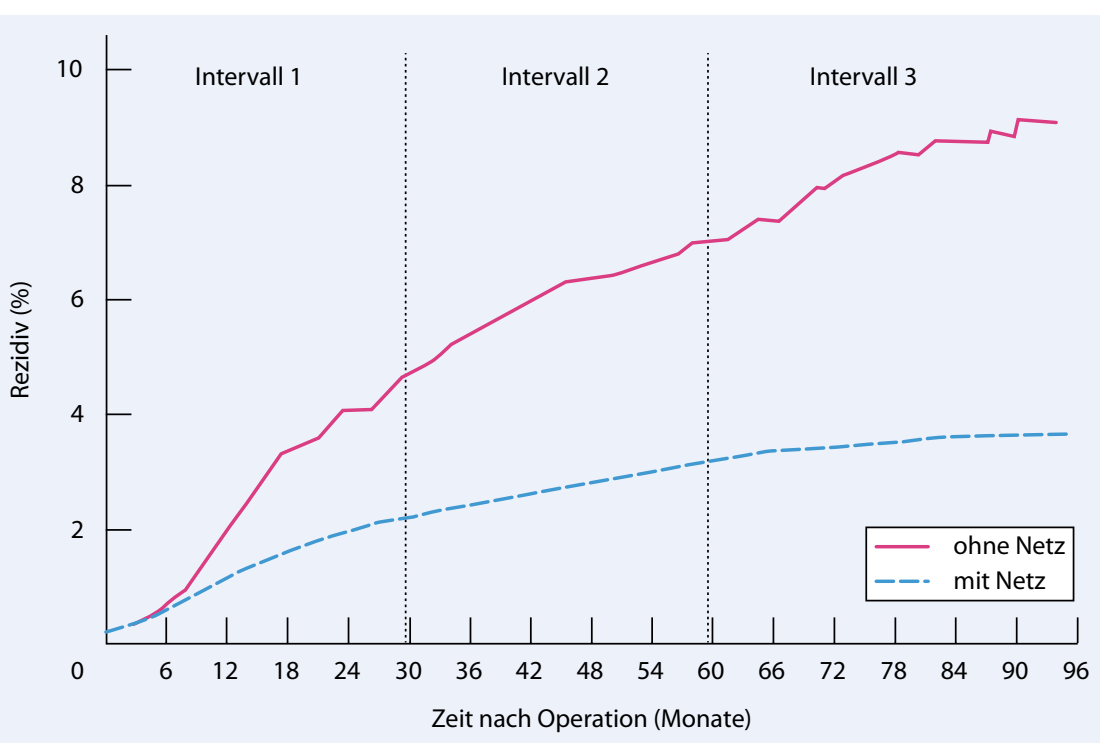

Abb. $1 \Delta$ Auftreten von Inguinalhernienrezidiven über die Zeit, Vergleich zwischen offener Hernienplastik mit Netz, respektive ohne Netz. Intervall 1: 0-30 Monate, Intervall 2: 30-60 Monate, Intervall 3: 60-96 Monate. (Adaptiert nach [3])

Als Mechanismus für den beeinträchtigten Wundheilungsprozess wird ein gestörter Kollageneinbau in die Wunde beschrieben. Vorstellbar ist auch eine vermehrte mechanische Belastung der Wunde durch chronischen Husten, welcher bei Rauchern häufig besteht.

Eine genetische Prädisposition ist für die Entwicklung von Bauchwandhernien relevant. So wurde eine positive Familienanamnese als deutlicher Risikofaktor für die Entwicklung einer primären Bauchwandhernie aufgezeigt [29]. Genexpressionsprofile von Patienten mit Narbenhernienrezidiven wurden mittels Polymerasekettenreaktion (PCR) und Microarray-Analysen untersucht. Es wurden dabei signifikante Unterschiede in der Genexpression von Haut- und Faszienzellen bei Patienten mit Rezidivhernien im Vergleich zur Kontrollgruppe gefunden [7]. Die Forschung bezüglich (Rezidiv-)Hernienentwicklung auf genetischer Ebene steht noch in den Anfängen, es ist zu hoffen, dass in diesem Bereich in den nächsten Jahren Erklärungen für die Unterschiede in der hereditären Prädisposition gefunden werden. Dies würde wiederum die gezielte Prophylaxe bei Hochrisikopatienten ermöglichen.

\section{Fazit für die Praxis}

- Der Erfolg einer Operationstechnik darf nicht nur an der Rezidivrate alleine gemessen werden, sondern muss alle für den Patienten relevanten Folgen (wie z. B. chronische postoperative Schmerzen) berücksichtigen.

- Indem das Rezidiv weniger Ausdruck der Patientenselektion als der Qualität der chirurgischen Technik ist, bleibt die Rezidivrate ein wichtiges Qualitätsmerkmal einer Hernienoperation.

- Bei der chirurgischen Therapie von Inguinalhernien werden mit den heutigen Techniken mittels laparoskopischer oder offener Netzeinlage sehr niedrige Rezidivraten erreicht. Bei einem Rezidiv nach einer Inguinalhernienoperation muss von einer chirurgischen Komplikation ausgegangen werden.

- Im Gegensatz zu Inguinalhernien liegt die Rezidivrate bei der Operation großer Narbenhernien trotz stetiger Optimierung der Technik weiterhin bei über $10 \%$. Bei sehr großen oder multiplen Hernien und zusätzlichen Risikofaktoren des Patienten kann eine Versorgung der Hernie sehr schwierig sein. Dem Patienten muss beim Aufklärungsgespräch erklärt werden, dass ein Rezidiv nicht selten ist.

- Hernienrezidive werden als Abweichung von der Norm wahrgenommen und hängen möglicherweise von der Indikation, der Wahl der Operation und der chirurgischen Technik und Taktik ab. Somit soll der Chirurg ein postoperatives Rezidiv in erster Linie als Komplikation und nicht als natürlichen Verlauf ansehen, mit dem Ziel seine Technik kontinuierlich zu verbessern.

\section{Korrespondenzadresse}

Prof. Dr. G. Beldi

Universitätsklinik für Viszerale Chirurgie und Medizin, Inselspital,

3010 Bern, Schweiz

guido.beldi@insel.ch

\section{Einhaltung ethischer Richtlinien}

Interessenkonflikt. A. Kohler und G. Beldi geben an, dass kein Interessenkonflikt besteht.

Dieser Beitrag beinhaltet keine Studien an Menschen oder Tieren.

\section{Literatur}

1. Andersen LP, Klein M, Gogenur l et al (2009) Longterm recurrence and complication rates after incisional hernia repair with the open onlay technique. BMC Surg 9:6

2. Birk D, Hess S, Garcia-Pardo C (2013) Low recurrence rate and low chronic pain associated with inguinal hernia repair by laparoscopic placement of $\mathrm{Pa}$ rietex ProGrip mesh: clinical outcomes of 220 hernias with mean follow-up at 23 months. Hernia 17:313-320

3. Bisgaard T, Bay-Nielsen M, Christensen IJ et al (2007) Risk of recurrence 5 years or more after primary Lichtenstein mesh and sutured inguinal hernia repair. Br J Surg 94:1038-1040

4. Bittner R, Kraft K, Schmedt CG et al (1998) Risks and benefits of laparoscopic hernia-plasty (TAPP). 5 years experiences with 3,400 hernia repairs. Chirurg 69:854-858

5. Bittner R, Leibl BJ, Kraft B et al (2011) One-year results of a prospective, randomised clinical trial comparing four meshes in laparoscopic inguinal hernia repair (TAPP). Hernia 15:503-510

6. Bittner R, Schwarz J (2012) Inguinal hernia repair: current surgical techniques. Langenbecks Arch Surg 397:271-282

7. Calaluce R, Davis JW, Bachman SL et al (2013) Incisional hernia recurrence through genomic profiling: a pilot study. Hernia 17:193-202 
8. Dietz UA, Winkler MS, Hartel RW et al (2012) Importance of recurrence rating, morphology, hernial gap size, and risk factors in ventral and incisional hernia classification. Hernia [Epub ahead of print]

9. Eker HH, Hansson BM, Buunen M et al (2013) Laparoscopic vs. open incisional hernia repair: a randomized clinical trial. JAMA Surg 148:259-263

10. Eklund AS, Montgomery AK, Rasmussen IC et al (2009) Low recurrence rate after laparoscopic (TEP) and open (Lichtenstein) inguinal hernia repair: a randomized, multicenter trial with 5-year followup. Ann Surg 249:33-38

11. Glassow F (1986) The Shouldice Hospital technique. Int Surg 71:148-153

12. Graham LA, Altom LK, Vick CC et al (2011) Agreement between patient survey and medical chart: Pitfalls in measurement strategies for hernia recurrence. Surgery 150:371-378

13. Helgstrand F, Rosenberg J, Kehlet H et al (2013) Nationwide prospective study of outcomes after elective incisional hernia repair. J Am Coll Surg 216:217-228

14. Helgstrand F, Rosenberg J, Kehlet $\mathrm{H}$ et al (2012) Reoperation versus clinical recurrence rate after ventral hernia repair. Ann Surg 256:955-958

15. Hull AJ (1913) Vl. Recurrence of Inguinal Hernia. Ann Surg 58:479-482

16. Kurmann A, Visth E, Candinas D et al (2011) Longterm follow-up of open and laparoscopic repair of large incisional hernias. World J Surg 35:297-301

17. Lichtenstein IL (1987) Herniorrhaphy. A personal experience with 6,321 cases. Am J Surg 153:553559

18. Nixon SJ, Jawaid H (2009) Recurrence after inguinal hernia repair at ten years by open darn, open mesh and TEP - no advantage with mesh. Surgeon 7:71-74

19. O'reilly EA, Burke JP, O'connell PR (2012) A metaanalysis of surgical morbidity and recurrence after laparoscopic and open repair of primary unilateral inguinal hernia. Ann Surg 255:846-853

20. Palmqvist E, Larsson K, Anell A et al (2013) Prospective study of pain, quality of life and the economic impact of open inguinal hernia repair. $\mathrm{Br} J$ Surg 100:1483-1488

21. Poobalan AS, Bruce J, Smith WC et al (2003) A review of chronic pain after inguinal herniorrhaphy. Clin J Pain 19:48-54

22. Sauerland S, Korenkov M, Kleinen T et al (2004) Obesity is a risk factor for recurrence after incisional hernia repair. Hernia 8:42-46

23. Schmedt CG, Sauerland S, Bittner R (2005) Comparison of endoscopic procedures vs Lichtenstein and other open mesh techniques for inguinal hernia repair: a meta-analysis of randomized controlled trials. Surg Endosc 19:188-199

24. Snyder CW, Graham LA, Vick CC et al (2011) Patient satisfaction, chronic pain, and quality of life after elective incisional hernia repair: effects of recurrence and repair technique. Hernia 15:123-129

25. Sorensen LT, Friis E, Jorgensen T et al (2002) Smoking is a risk factor for recurrence of groin hernia. World J Surg 26:397-400

26. Sorensen LT, Hemmingsen UB, Kirkeby LT et al (2005) Smoking is a risk factor for incisional hernia. Arch Surg 140:119-123

27. Tse GH, Stutchfield BM, Duckworth AD et al (2010) Pseudo-recurrence following laparoscopic ventral and incisional hernia repair. Hernia 14:583-587

28. Van Veen RN, Wijsmuller AR, Vrijland WW et al (2007) Randomized clinical trial of mesh versus non-mesh primary inguinal hernia repair: longterm chronic pain at 10 years. Surgery 142:695698
29. Zoller B, Ji J, Sundquist J et al (2013) Shared and nonshared familial susceptibility to surgically treated inguinal hernia, femoral hernia, incisional hernia, epigastric hernia, and umbilical hernia. J Am Coll Surg 217:289-299 e281

Kommentieren Sie diesen Beitrag auf springermedizin.de

- Geben Sie hierzu den Beitragstitel in die Suche ein und nutzen Sie anschließend die Kommentarfunktion am Beitragsende.

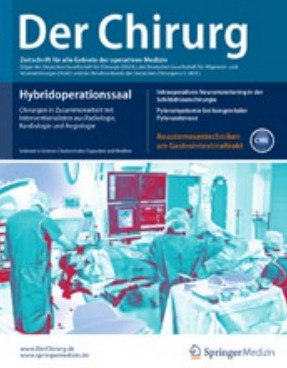

2.,Julius-Springer-Preis für Chirurgie" gestiftet von SpringerMedizin

Der 2013 ins Leben gerufene „JuliusSpringer-Preis für Chirurgie" wird auch im kommenden Jahr von Springer Medizin verliehen. Der mit 3000 EUR dotierte Preis soll die wissenschaftliche Forschung auf dem Gebiet der Chirurgie unterstützen und insbesondere den Nachwuchs fördern. Prämiert wird die beste frei eingereichte Arbeit der Rubriken „Originalien" oder „Übersichten", die im Jahr 2013 in Der Chirurg publiziert wurde. Beurteilt werden die Beiträge durch die Schriftleitung und das Herausgeberboard von Der Chirurg. Als Anerkennung und Wertschätzung der Leistungen unserer Autoren wird zudem im Rahmen eines festlichen Abends im Zuge des DGCH-Kongresses 2014 der Leser-Preis für die jeweils herausragendste Arbeite der Rubriken "Leitthema" und "CME Zertifizierte Fortbildung" vergeben.

Möchten auch Sie eine „Übersicht“ oder "Originalie“ für Der Chirurg einreichen?

Um Ihnen bei der Manuskripterstellung behilflich zu sein, haben wir für unsere Autoren ausführliche Autorenleitfäden und Musterbeiträge zusammengestellt. Diese und weitere Hinweise zur Manuskripterstellung finden Sie auf www.DerChirurg.de unter dem Menüpunkt "Hinweise für Autoren".

Bitte reichen Sie Ihren fertigen Beitrag über unser online Begutachtungssystem Editorial Manager ein: http://dech.edmgr.com/

Wir freuen uns auf Ihre Beiträge!

Ihre Redaktion Der Chirurg 